\title{
Article
}

\section{The importance of significant event analysis}

Cooper-Moss, Nicola, Smith, Neil and Chauhan, Umesh

Available at http://clok.uclan.ac.uk/34231/

Cooper-Moss, Nicola, Smith, Neil and Chauhan, Umesh ORCID: 0000-00020747-591X (2020) The importance of significant event analysis. InnovAiT, 13 (10). ISSN $1755-7380$

It is advisable to refer to the publisher's version if you intend to cite from the work. http://dx.doi.org/10.1177/1755738020941231

For more information about UCLan's research in this area go to http://www.uclan.ac.uk/researchgroups/ and search for <name of research Group>.

For information about Research generally at UCLan please go to http://www.uclan.ac.uk/research/

All outputs in CLoK are protected by Intellectual Property Rights law, including Copyright law. Copyright, IPR and Moral Rights for the works on this site are retained by the individual authors and/or other copyright owners. Terms and conditions for use of this material are defined in the policies page.

\section{CLoK}

Central Lancashire online Knowledge www.clok.uclan.ac.uk

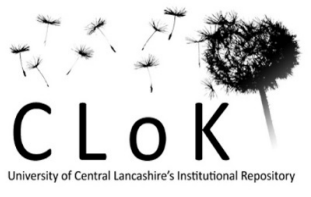




\section{The importance of Significant Event Analysis}

\begin{tabular}{|r|l|}
\hline Journal: & InnovAiT \\
\hline Manuscript ID & Draft \\
\hline Manuscript Type: & Non-Clinical Article \\
\hline Manuscript Keywords: & Cancer, Clinical Governance, Patient Safety \\
\hline & \\
\end{tabular}

\section{SCHOLARONE Manuscripts}




\section{The importance of Significant Event Analysis}

Significant Event Analysis (SEA) is a structured quality improvement activity that is well established in General Practice. Participation in SEA prompts primary care teams to reflect on their clinical reasoning; highlight exemplary care and identify any potential improvements in both practice and wider health-care systems. This article provides an overview of the SEA process and the events surrounding a SEA meeting. Cancer care examples are used to demonstrate how SEA can be optimised to enhance team-based learning and improve future patient care.

\section{The RCGP curriculum and Significant Event Analysis (SEA)}

The role of the General Practitioner is to:

- Recognise how the analysis of patient safety incidents can enhance rather than undermine professional integrity and performance.

- Understand how and when to apply tools to improve quality of care.

- Engage in significant event reviews, in a timely and effective manner.

- Contribute to regular SEA meetings and recognise the benefits of a multidisciplinary team.

- Feed-back to colleagues about incidents.

This article will cover the following areas:

- Background and classification of significant events

- The SEA process

- SEAs of new cancer diagnoses

- Benefits and barriers to SEA

- Wider implications for system-based change

\section{Background and classification of significant events}

Case review is a time-honored learning technique, which has been traditionally applied to advance understanding of illness and disease. Recent years have shown increasing 
recognition of case-based analysis as an opportunity to identify potential improvements in patient care. The General Medical Counsel (GMC) states in Good Medical Practice that doctors must contribute to adverse event recognition to help keep patients safe. A significant event is defined as "any event thought by anyone in the team to be significant in the care of patients or the conduct of the practice" (Pringle et al, 1995). The event of interest may be adverse, exemplary or simply important, for example, a new cancer diagnosis. Team-based analysis of significant events has evolved from a human factors approach; examining the complex interactions between people, activity, and the wider environment (The Health Foundation, 2016). Box 1 provides a list of potential examples of SEA topics.

SEA meetings may involve anyone in the practice team including clinicians, practice pharmacists, receptionists, administrative staff and practice managers. Involvement in quality improvement activities, such as SEA, is now considered to be an integral component of continuing professional development and reflection for individuals and practice teams. Evidence of participation in the case review process is necessary for satisfactory progression in General Practitioner (GP) appraisals and revalidation.

\section{(insert box 1 here)}

The events which are highlighted through the SEA process may involve patient safety incidents or "near-misses". These events can be categorised according to the severity of patient harm; including no harm, low, moderate, severe or death (The National Patient Safety Agency, 2005). The severity of patient harm can be attributed to the impact on the patient's physical, mental or social functioning; as well as the level of resultant intervention required.

\section{The SEA process}

The SEA process can be considered in two parts: 1) the events surrounding a SEA meeting, and 2) the components of a SEA meeting. 


\section{The events surrounding a SEA meeting}

The SEA process extends beyond the SEA meeting itself. Prior to the meeting, the "significant event" should be identified, prioritised and relevant data should be collected. The event may be highlighted by a team member who has been involved in or witnessed the event; through patient feedback or a complaint. Patients can be made aware of the SEA process through waiting room posters and practice leaflets. Patient data should be anonymised and patient confidentiality should be protected, particularly if information is submitted for the purposes of local clinical audit. It is good practice to make patients aware that their data may be used in this way; giving them an opportunity to object (Medical Defense Union, 2017).

Each SEA meeting should be logistically organised to involve relevant team members according to each individual case. For example, events surrounding cancer care may involve specific health professionals such as Macmillan nurses, district nurses and community palliative care professionals. The actions to be taken from the meeting should be documented, with corresponding implementation and review dates. Team discussions may highlight additional significant events which may form the subjects of subsequent meetings. A written summary of the SEA discussion and action points may also be offered to the patient(s) involved. Patients may also be invited to a debrief meeting for feedback or as part of a complaint resolution procedure.

\section{The components of a SEA meeting}

Although different practices may adopt various flexible approaches to SEA, there are four main components of a SEA discussion which are commonly described in the literature:

- the event process (what happened?)

- reflection (why did it happen?)

- learning (what has been learned?)

- recommendations (what has or could be changed?)

Reflections may include positive aspects of care; areas for improvement and degrees of severity of harm. Each case may highlight immediate management, prevention, 
follow-up and/or wider organisational concerns. In addition, the SEA process can be utilised to consider the impact on the individuals involved and reflection on what was effective about each SEA (Royal College of General Practitioners, 2016). The learning and recommendations form the basis of practice action plans; the progress of which can be reviewed at subsequent SEA meetings. These steps are summarised in Figure 1.

(Insert figure 1 here)

\section{SEA of new cancer diagnoses}

SEAs involving a delay in a significant diagnosis, such as a new cancer diagnosis, can help in understanding complex diagnostic processes and referral pathways. GPs play an important role in the early diagnosis of cancer, as patients commonly present to them with early or vague symptoms. Despite this, more than one in five cancers in England are diagnosed during an emergency admission; with an associated reduction in survival. With this in mind, the 2015-2020 national cancer strategy has made the specific recommendation that all General Practices should undertake annual SEAs of such cases (Independent Cancer Taskforce, 2015). Boxes 2 and 3 present fictional case examples of new cancer diagnoses from a SEA meeting in General Practice. The recommendations may relate to patients, practice teams, secondary care and local Clinical Commissioning Groups (CCGs).

(Insert box 2 here)

(Insert box 3 here)

\section{Benefits and barriers to SEA}

\section{Benefits}

Participation in SEA meetings provides an opportunity for shared learning and celebration of good care within General Practices. How we learn from and share lessons regarding clinical care is an important marker of our personal and collective 
professional development. SEAs provide evidence of learning at an individual level for GP appraisals and at a practice level for Care Quality Commission (CQC) reviews. If applied rigorously, SEA can be utilised to learn from a wide variety of quality issues including those resulting in patient harm.

SEA combines factual events with subjective judgements or experiences, which often carry an emotional weight. This emotional involvement has the potential to enhance learning and bring about change in clinical practice. SEA encourages individual practices to develop team-working strategies and adopt a structured approach for the effective implementation of action plans. In addition, thematic analysis of multi-site SEA reports on cancer cases has been shown to provide valuable insights into diagnostic processes and care pathways from a primary care perspective (Mitchell et al, 2013, 2015).

\section{Barriers}

Although SEA is widely accepted as a feasible tool for quality improvement in primary care, engagement among GPs and individual practices is variable. The beneficial emotional impact described previously may also be viewed as a barrier for participation for some individuals. The SEA process can be disconcerting, particularly for clinicians, as missed or delayed diagnoses are uncovered. Engagement may be influenced by concerns regarding confidentiality, litigation, or professional embarrassment. Furthermore, there may be difficulty determining when an event is "significant" and uncertainty regarding the SEA process among different team members.

Individual GPs may also feel restricted in their ability to influence change within wider health care systems. They may feel empowered to make internal changes within their practice but not, for example, be able to obtain access to additional diagnostics or modify hospital activities. Effective leadership is required to ensure learning and recommendations from SEA discussions are disseminated and action plans are effectively implemented. There are also a number of financial, time and training implications involved for practices upholding regular SEA meetings. 


\section{Wider implications for system-based change}

Significant events are rarely simply the result of one person's actions or inactions. Learning can often be translated into system-level recommendations. It is therefore important to move away from a culture which attributes individual blame and towards a constructive system-focused approach. Multi-site recommendations from practice SEA meetings provide Clinical Commissioning Groups (CCGs) with emerging themes for local quality improvement; informing future resource provision and re-design of local referral pathways. These may form the basis of multi-disciplinary educational events and quality improvement workshops.

A review of SEA reports in primary care concluded that although SEA often results in practice reported changes, the long-term sustainment of improvement in patient care is unclear (Mackay et al, 2009). For genuine impact there needs to be consensus on key priorities and improvements in patient pathways and services. This may require extra funding, new staff and piloting of fresh ideas. Further research is required into the factors affecting system-level change following SEA and the impact on the wider indicators for quality of care.

\section{Conclusions}

Significant Event Analysis is a feasible and acceptable quality improvement activity which provides multiple benefits for the individuals and teams involved. Individual practices may experience certain barriers for optimal participation in the SEA process, however, these can be overcome through effective team working, training and leadership. Engagement in SEA is an ideal opportunity for both GP trainees and early career GPs to share their concerns, exercise leadership, and influence change within their practice and wider healthcare systems. 


\section{Key points}

- Significant Event Analysis (SEA) is a flexible method for learning from a wide range of clinical and non-clinical events.

- A structured team-based approach to SEA optimises collective learning and successful implementation of action plans.

- GPs should consider any barriers to effective SEA within their practice; encouraging a supportive environment for reflection.

- SEA is a recognised educational tool for learning from new cancer cases. Practices are supported by the RCGP cancer SEA toolkit.

- GPs are in a strong position to influence improvements within their practice and wider healthcare community.

- Findings from multi-site SEAs can inform local commissioning intentions and improvements in patient care pathways.

\section{References and further information}

Independent Cancer Taskforce (2015) Achieving world-class cancer outcomes: a strategy for England 2015-2020.

McKay J, Bradley N, Lough M, et al. (2009) A review of significant events analysed in general practice: implications for the quality and safety of patient care. BMC Fam Pract 10(61): 1471-2296.

Medical Defense Union (2017) Significant Event Analysis. Available at: https://www.themdu.com/guidance-and-advice/guides/guide-to-significant-eventanalysis (accessed at 23 October 2017).

Mitchell ED, Rubin G, Macleod U (2013) Understanding diagnosis of lung cancer in primary care: qualitative synthesis of significant event audit reports. $\mathrm{Br} \mathrm{J}$ Gen Pract. 63:37-46. 
Mitchell ED, Rubin G, Merriman L et al (2015) The role of primary care in cancer diagnosis via emergency presentation: qualitative synthesis of significant event reports. Br J Cancer. 112:50-56.

Pringle M, Bradley CP, Carmichael CM et al. (1995) Significant Event Auditing. Occasional Paper 70. Exeter: Royal College of General Practitioners.

Royal College of General Practitioners (2016) Significant Event Audit (SEA) of Cancer Diagnosis Template. London: RCGP.

Royal College of General Practitioners (2019) Early Diagnosis of Cancer Significant Event Analysis Toolkit. Available at: https://www.rcgp.org.uk/clinical-andresearch/resources/toolkits/early-diagnosis-of-cancer-significant-event-analysistoolkit.aspx.

The Health Foundation (2016) Four tools to enhance significant event analysis in primary care. Available at: https://www.health.org.uk/newsletter-feature/four-tools-toenhance-significant-event-analysis-in-primary-care (accessed 12 September 2019).

The National Patient Safety Agency (2005) Seven steps to patient safety for primary care. London: NPSA.

\section{Conflict of interest}

The authors are involved in the implementation and analysis of multi-site significant event analyses of cancer cases across General Practices within their locality. 


\section{Box 1: Examples of SEA topics}

- New cancer diagnoses

- Preventative care e.g. missed opportunities for vaccination

- Chronic disease management e.g. diabetes

- Prescribing errors

- Suicide attempts

- Unexpected death

- Non-accidental injury

- Emergency situations e.g. peri-arrest, acute asthma exacerbation

- Confidentiality breach

- Organisational e.g. appointment system, delayed referrals 
Figure 1: Components of a SEA meeting

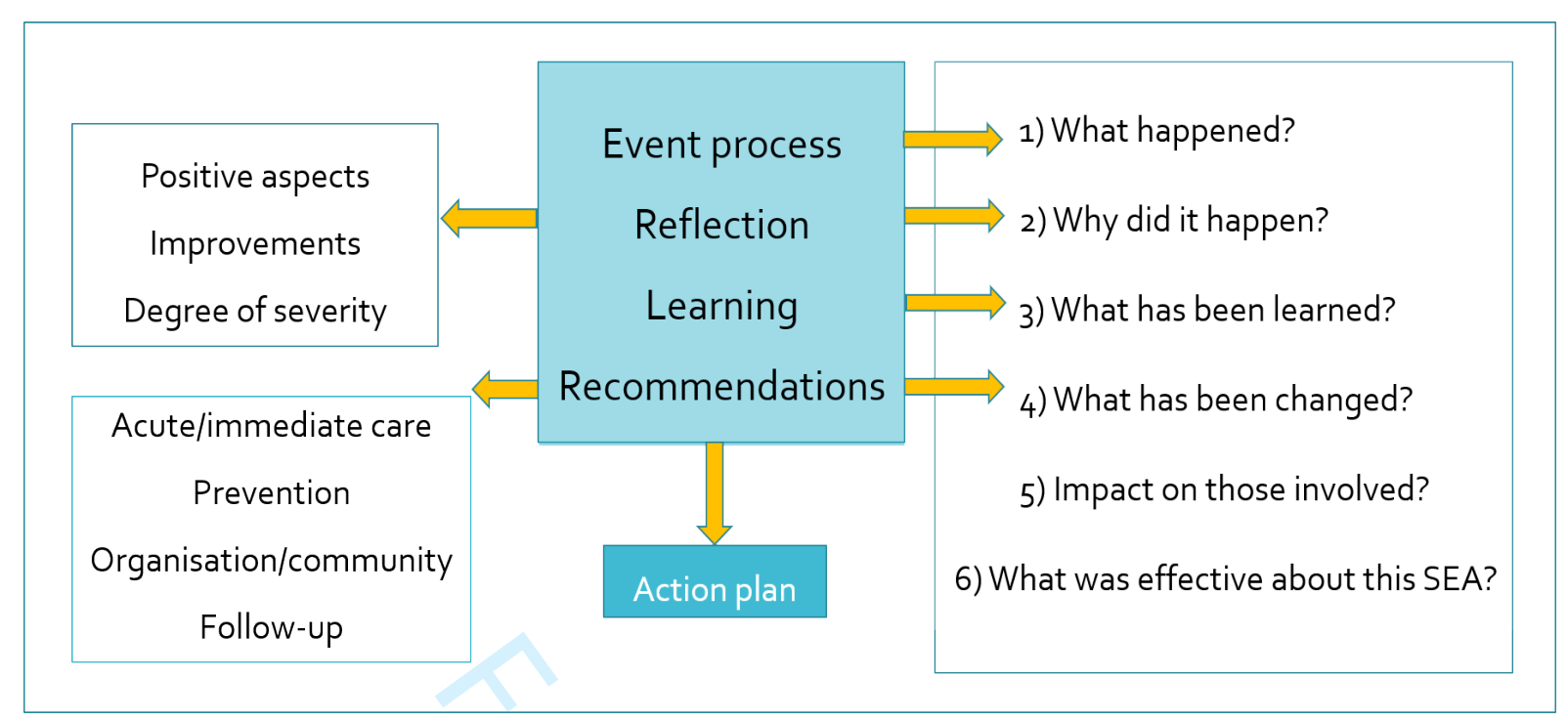




\section{Box 2: SEA meeting for case example Mr A}

Mr A is a 65-year-old male who has been diagnosed with palliative lung cancer. $\mathrm{He}$ is an ex-smoker and suffers from Chronic Obstructive Pulmonary Disease (COPD), depression and anxiety. He has seen the practice nurse and various GPs on a number of occasions with reports of increasing breathlessness, poor appetite and weight loss over the last twelve months. A chest X-ray was reported as "normal" six months ago. He has now been admitted with hemoptysis. A CT scan has revealed metastatic lung cancer.

\section{Event process: what happened?}

- Delay in lung cancer diagnosis

- Late stage emergency presentation

\section{Reflection: why did it happen?}

- Missed opportunities for prevention: smoking cessation

- Diagnostic over-shadowing with pre-existing co-morbidities

- Lack of continuity of care and escalation of 'red flag' symptoms

- False negative chest X-ray

\section{Learning: what has been learned?}

- Importance of patient education and smoking cessation support

- Recognition and follow-up of non-resolving or worsening symptoms

- Diagnostic limitations of chest X-ray for lung cancer

- High index of suspicion with smoking history and/or COPD

\section{Recommendations: what has been changed}

- Patient education through waiting room posters/advertising

- Practice education event on the presentations of lung cancer

- Protocol for re-attenders with non-resolving symptoms

- Practice escalation pathway from nurse-led COPD clinic

- Read-coding of patient symptoms and safety-netting advice

- Respiratory liaison system and improved radiology access 


\section{Box 3: SEA meeting for case example Mrs B}

Mrs $B$ is a 70-year-old female who has been diagnosed with colon cancer. She presented with a six-month history of lower abdominal pain and bloating. Blood tests revealed a mildly raised serum CA125. She was referred urgently to gynecology, but was discharged following a normal ultrasound scan. Mrs B did not attend a follow-up appointment at the practice as she presumed it was no longer required. She was admitted to hospital nine months later with bowel obstruction secondary to colorectal cancer.

\section{Event process: what happened?}

- Delay in bowel cancer diagnosis

- No follow-up following initial suspected cancer referral

Reflection: why has it happened?

- Missed bowel cancer screening opportunities

- Appropriate initial suspected cancer referral

- Gynecology discharge letter filed without clinician review

Learning: what has been learned?

- Importance of patient education and safety-netting

- Follow-up of ongoing symptoms despite negative investigations

- Use of the fecal immunochemical test (FIT)

Recommendations: what has been changed?

- Practice audit of bowel cancer screening uptake - contact of patients

- Multi-disciplinary education event on the presentations of bowel cancer, referral pathways and the fecal immunochemical test (FIT)

- Protocol for follow-up of suspected cancer referrals / non-attenders

- Review of inter-departmental secondary care suspected cancer referrals 